\title{
MODULATION CHARACTERISTICS FOR PARAMETRIC RECEIVING ARRAYS
}

\author{
James J. TRUCHARD
}

Applied Research Laboratories The University of Texas at Austin Austin, Texas 78712, U.S.A.

Résumé. - Lors d'études expérimentales de rêseaux paramétriques de réception on a utilisé un traitement du signal par démodulation de phase et d'amplitude. Les expressions théoriques des composantes de bande latérale aux fréquences somme et différence ont été obtenues à partir de l'équation d'onde du second ordre, due à Westervelt. Ces composantes ont été additionnées ã la porteuse puis exprimées comme composantes de modulation de celle-ci. Pour les expériences on a utilisé un rếseau paramëtrique de $15 \mathrm{~m}$, fonctionnant à $90 \mathrm{kHz}$, avec des fréquences de signal de 3 à $6 \mathrm{kHz}$. Les signaux d'entrée de l'hydrophone ont été filtrês au moyen d'un filtre de bande et ecrêtés pour s'assurer de la suppression de toute modulation d'amplitude, et la modulation de phase du signal a été mesurée à l'aide d'un détecteur de phase.

Les diagrammes de rayonnements mesurês à la sortie du détecteur de phase restent inchangēs lors du désalignement de la pompe ou de l'hydrophone dans le réseau, par contre les composantes de modulation d'amplitude sont considérablement modifiées. Ce résultat offre un bon accord avec les calculs théoriques. La théorie et 1 'expérience montrent clairement que la modulation d'un son de fréquence élevée par une onde sonore de basse fréquence est essentiellement une modulation de phase.

Abstract. - Experiments have been conducted on the parametric receiving array using amplitude and phase demodulators for signal processing. Theoretical expressions were derived by finding the sum and difference frequency sideband components using the second-order wave equation originated by Westervelt. The sum and difference frequency components were added to the carrier and then expressed in terms of modulation components of the carrier. Experiments were conducted using a $15 \mathrm{~m}$ parametric receiving array operating at $90 \mathrm{kHz}$. Signal frequencies in the renge from 3 to $6 \mathrm{kHz}$ were used. The hydrophone input signals were bandpass filtrered and then clipped to ensure that no amplitude modulation was left on the signal. A phase detector was used to observe the phase modulation of the signal. Beam patterns measured at the phase detector output were unchanged when either the pump or the hydrophone in the array was misaligned. On the other hand, the amplitude modulation components changed dramatically when the pump or the hydrophone was misaligned. This result agreed well with the theoretical expressions. The theory and the experiment clearly demonstrate that the modulation of a high frequency sound wave by a low frequency sound wave is primarily a phase modulation.

1. INTRODUCTION. - In previous papers, /1-3/ the author studied the sideband characteristics of the parametric receiving array signal. Theoretical expressions were derived for the sum and the difference frequency components for several geometries for the parametric receiving array. The amplitude of each sideband signal was measured and compared to the theoretical expressions. The theory agreed well with the experimental results in both the case of aligned and misaligned transducers. The experiments, however, did not measure the relative phase between the carrier and the two sideband signals. In this paper, the theory is formulated in a form which can be used to verify experimentally the phase characteristics of the sideband signals. In order to accomplish this, the modulation processes are described in terms of amplitude and phase modulations. The theoretical results which were previously obtained in Ref. 2 are reworked into a form which gives the expression for the amplitude and the phase modulation caused by the modulation process.
Since the phase of the two sideband signals is related to the type of modulation, we have a means to experimentally measure the phase characteristics of the sideband signals by measuring the amplitude and phase modulation components of the signa1. In this paper, we describe some experiments which were conducted using amplitude and phase demodulators for the measurement of the modulation process. We find that expressions formulated in the previous paper, when expressed in terms of amplitude and phase modulation terms, do agree well with the actual experimental results.

\section{MODULATION CHARACTERISTICS FOR CASES OF THE} PARAMETRIC RECEIVING ARRAY. - We will now reconsider the parametric receiving array with an omnidirectional pump. The geometry for this case is given Fig. 1. The signal source is assumed to be far from the parametric receiver so that the low frequency waves are approximately planar in the vicinity of the receiver. Then at the point $(X, Y, Z)$ 
and at time $t$, the sound waves can be represented in complex form as

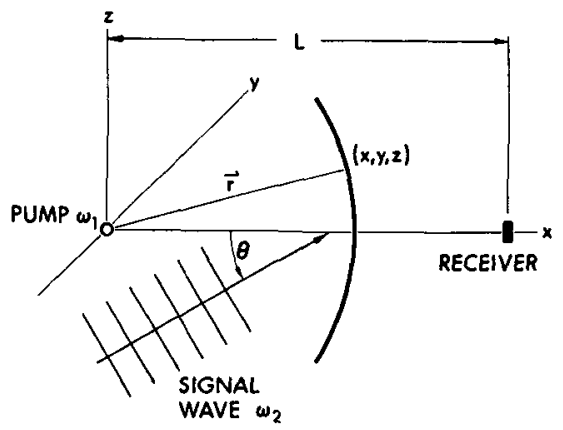

Fig. 1. - Parametric Receiving Array with a point Source Pump.

$p_{11}=j \frac{P_{11}}{L} \exp \left[-\left(a_{1}-j k_{1}\right) L-j \omega_{1} t\right]$

and

$p_{12}=P_{12} \exp \left[-\left(\alpha_{2}-j k_{2}\right)(x \cos \theta+y \sin \theta)-j \omega_{2} t\right],(2)$

where

$L=\left(x^{2}+y^{2}+z^{2}\right)^{1 / 2}$.

We found the second order pressure to be

$p_{2}=\bar{p} \exp \left[\bar{\mp} \frac{j k_{2} L(1-\cos \theta)}{2}\right](\sin M) / M$,

where

$M=k_{2} L(1-\cos \theta) / 2$

and

$\bar{p}=-\frac{\omega_{ \pm} 1+\frac{B}{2 A}}{2 p_{0} c_{0}^{3}} P_{11} P_{12} \exp \left[-\alpha_{ \pm} L+j\left(k_{ \pm} L-\omega_{ \pm} t\right)\right]$.

In this example, the carrier is $p_{11}$ and the sidebands are $p_{2}(+)$ and $p_{2}(-)$. If we sum the three signals together and consider only the real terms, we have

$$
\begin{aligned}
p= & -P_{11} \frac{\exp \left[-\alpha_{1} L\right] \sin (X)}{L} \\
& -\frac{\left(\omega_{1}+\omega_{2}\right)}{2} \frac{\beta}{[} P_{11} \exp \left[-\alpha_{1} L\right] \cos [X+\gamma] \\
& -\frac{\left(\omega_{1}-\omega_{2}\right)}{2} \frac{\beta}{[} P_{11} \exp \left[-{ }_{1} L\right] \cos [X+Y],
\end{aligned}
$$

where

$$
\begin{aligned}
& X=k_{1} L-\omega_{1} t, \\
& Y=k_{2} L-\omega_{2} t-M,
\end{aligned}
$$

and

$$
\beta=L \frac{1+\frac{B}{2 A}}{\rho_{0} C_{0}^{3}} p_{12} \frac{\sin M}{M} \text {, }
$$

and we let $\alpha_{ \pm}=\alpha_{1}$ since $\omega_{ \pm} \approx \omega_{1}$.

The pressure can then be expressed in the form

$p=\frac{-P_{11} \exp \left[-a_{1} L\right]}{L}$

$\left[\sin X+\omega_{1} \beta \cos X \cos Y-\omega_{2} \beta \sin X \sin Y\right]$.

Next, we express the pressure in terms of amplitude and phase modulation components by making use of the approximations

$$
\sin \left(\omega_{1} \beta \cos Y\right) \cong \omega_{1} \beta \cos Y,
$$

and

$$
\cos \left(\omega_{1} \beta \cos \gamma\right) \approx 1,
$$

since $\left|\omega_{1} \beta \cos Y\right| \ll 1$. Using the identity $[\sin (C+D)=\sin C \cos D+\cos C \sin D]$, we can express the pressure as

$$
\begin{aligned}
p= & \frac{-P}{L} \exp \left[-\alpha_{1} L\right] \\
& {\left[\sin \left(X+\omega_{1} \beta \cos Y\right)-\omega_{2} \beta \sin X \sin Y\right] . }
\end{aligned}
$$

We can see that the pressure has two components : one that represents a phase modulation and one that represents an amplitude modulation. We also see that the phase modulation is proportional to $\omega_{1}$ while the amplitude modulation is proportional to $\omega_{2}$. Since $\omega_{1} \gg \omega_{2}$ for the parametric receiving array, the phase modulation term will dominate.

In our next example we consider a line pump source and a point receiver. The geometry for this example is schown in Fig. 2.

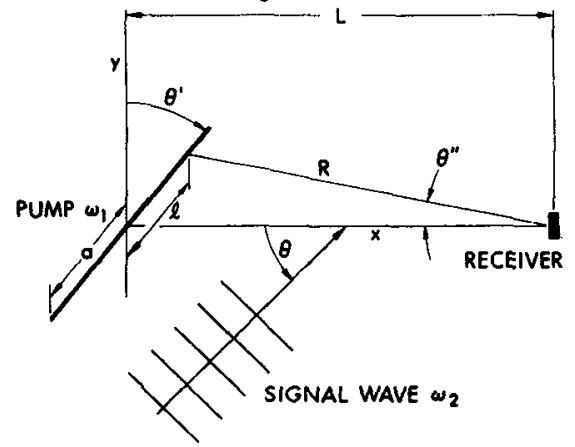

Fig. 2. - Parametric Receiving Array with a Line Source Pump. 
The sideband pressure for the sum and difference frequency components was found to be $\mathrm{e}^{1-3}$

$p_{2}=\bar{p} \exp ( \pm M)\left[\frac{\sin M}{M}\right]\left[\frac{\operatorname{sinaA_{\pm }}}{\mathrm{a} A_{ \pm}}\right]$,

where

$A_{ \pm}= \pm \frac{1}{2} k_{2} \sin \left(\theta+\theta^{\prime}\right) \pm k_{2} \sin \theta^{\prime}-k_{ \pm} \sin \theta^{\prime}$,

and $\bar{p}$ is the same as Eq. 6 .

Including the usual expression for the farfield of a line array, the total pressure in the farfield of the line source is

$$
\begin{aligned}
p & =\frac{-P_{11} \exp \left[-\alpha_{1} L\right]}{L} \sin x\left(\frac{\sin \left(k_{1} a \sin \theta^{\prime}\right)}{k_{1}{ }^{a} \sin \theta}\right) \\
& -\frac{\left(\omega_{1}+\omega_{2}\right)}{2} \hat{\beta} P_{11} \exp \left[-\alpha_{1} L\right] \cos [X+\gamma]\left[\frac{\left(\sin A_{ \pm}\right)}{a A_{+}}\right] . \\
& -\frac{\left(\omega_{1}-\omega_{2}\right)}{K} P_{11} \exp \left[-\alpha_{1} L\right] \cos [X-\gamma]\left[\frac{\left(\sin A_{-}\right)}{a A_{-}}\right] .
\end{aligned}
$$

Expanding the cosines and rearranging we get

$$
\begin{aligned}
p & =\frac{-P_{11} \exp \left[-\alpha_{1} L\right]}{L}\left[\frac{\sin \left(k_{1} a \sin \theta^{\prime}\right)}{k_{1} a^{2} \sin \theta^{T}} \sin X\right. \\
& -\left[\frac{\omega_{1} B}{2}\left(\frac{\sin A_{+}}{a A_{+}}+\frac{\sin a A_{-}}{a A_{-}}\right)\right. \\
& \left.+\frac{\omega_{2} B}{2}\left(\frac{\sin a A_{+}}{a A_{+}}-\frac{\sin a A_{-}}{a A_{-}}\right)\right] \cos X \cos Y \\
& +\left[\frac{\omega_{1} B}{2}\left(\frac{\sin a A_{+}}{a A_{+}}-\frac{\sin a A_{-}}{a A_{-}}\right)\right. \\
& \left.\left.+\frac{\omega_{2} B}{2}\left(\frac{\operatorname{sinaA_{+}}}{a A_{+}}+\frac{\sin a A_{-}}{a A_{-}}\right)\right] \sin X \sin Y\right] .
\end{aligned}
$$

Remembering that $\beta \omega_{1} \ll 1, \omega_{2} \ll \omega_{1}$, we can express this in the following approximate form :

$$
\begin{aligned}
p=-P_{11} \frac{\exp \left[-\alpha_{1} L\right]}{L} \frac{\sin \left(k_{1} a \sin \theta^{\prime}\right)}{k_{1} a \sin \theta^{\prime}} \\
\quad \times\left[\sin \left(k_{1} L-\omega_{1} t-\phi(t)\right)+A(t) \sin \left(k_{1} L-\omega_{1} t\right)\right],
\end{aligned}
$$

where

$$
\begin{aligned}
\phi(t) & =\frac{B\left(k_{1} a \sin \theta^{\prime}\right)}{2 \sin \left(k_{1} a \sin \theta^{\top}\right)} \cos \left(k_{2} L_{-}-\omega_{2} t-M\right) \\
& \times\left[\omega_{1}\left(\frac{\sin A_{+}}{a A_{+}}+\frac{\sin a A_{-}}{a A_{-}}\right)+\omega_{2}\left(\frac{\sin A_{+}}{a A_{+}}-\frac{\sin a A_{-}}{a A_{-}}\right)\right] .
\end{aligned}
$$

and

$$
\begin{aligned}
A(t) & \left.=\frac{B\left(k_{1} a \sin \theta^{\prime}\right)}{2 \sin \left(k_{1} a \sin \theta\right.}\right) \sin \left(k_{2} 1-\omega_{2} t-M\right) \\
& \times\left[\omega_{1}\left(\frac{\sin a A_{+}}{a A_{+}}-\frac{\sin A_{-}}{a A_{-}}\right)+\omega_{2}\left(\frac{\sin A_{+}}{a A_{+}}+\frac{\sin A_{-}}{a A_{-}}\right)\right] .
\end{aligned}
$$

Since the carrier frequency is much greater than the signal frequency, the first term in Eq. (18) will be many times larger than the second term. The term sinaA ${ }_{+} / a A_{+}-\operatorname{sina} A_{-} / a A_{-}$will also be very small if the receiver is on the axis of the pump transducer. Therefore, for a 11 practical purposes, the second term in Eq. (18) can be ignored. We then have the phase modulation component to be

$$
\begin{aligned}
\phi(t)= & \frac{\omega_{1} B\left(k a \sin \theta^{\prime}\right)}{2 \sin \left(k a \sin \theta^{T}\right)} \\
& \left(\frac{\operatorname{sina} A_{+}}{a A_{+}}+\frac{\sin a A_{-}}{a A_{-}}\right) \cos \left(k_{2} L-\omega_{2} t-M\right) .
\end{aligned}
$$

Both terms in the amplitude modulation expression will be smal1 because, in general, sina $A_{+} / a A_{+}$- sina $A_{-} / a A_{-}$will be a small term even when multiplied by $\omega_{1}$. The second term is proportional to $\omega_{2}$ and will be very small also. Consequently, for most practical cases of parametric receiving arrays, the phase modulation component will be substantially larger than the amplitude modulation component. The expression sina $A_{+} / a A_{+}+\operatorname{sina} A_{-} / a A_{-}$will remain essentially constant as the angle of the pump transducer is changed slightiy. As a consequence, the beam pattern for the phase modulation term will remain essentially unchanged if we misalign the pump transducer. On the other hand, the amplitude modulation component will be significantly changed as we misalign the pump transducer because it includes a term which is zero when the pump transducer is aligned, but nonzero when the pump transducer is misaligned. As a consequence, the beam patterns for the phase modulation term and the amplitude modulation term will be substantially different when the pump transducer is misaligned. This charasteristic can be easily verified experimentally.

EXPERIMENTS. - A series of experiments was conducted to verify the characteristics which we have studied in the previous section. A pictorial of 


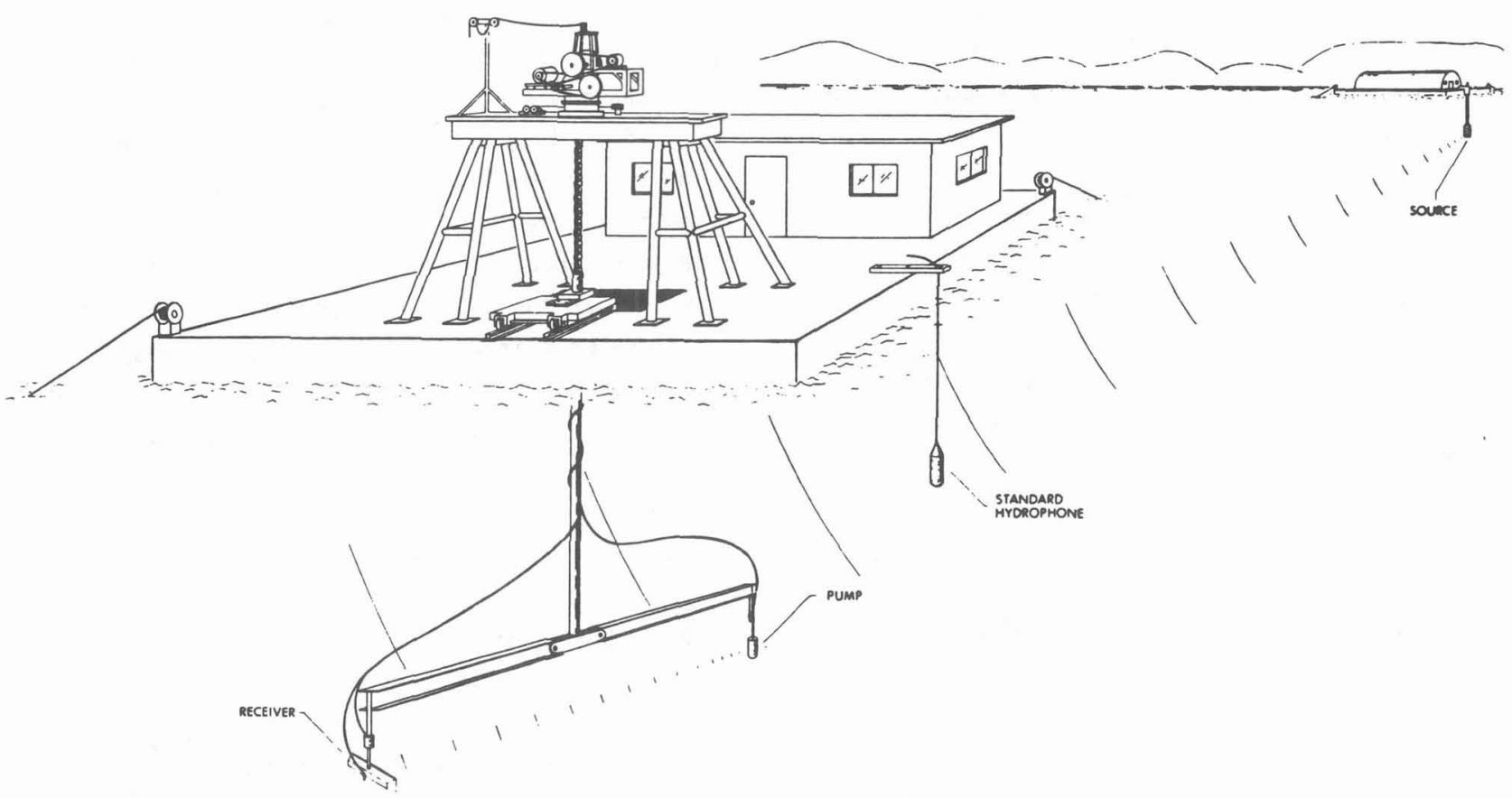

Fig. 3. - Pictorial for the Parametric Receiving Array Experiment

the experiment is shown in Fig. 3. The parametric receiving array is described in ref. 3 . The pump transducer, which is either a point source or line source, generated a $90 \mathrm{kHz}$ carrier frequency which is received by a second transducer $15 \mathrm{~m}$ from the

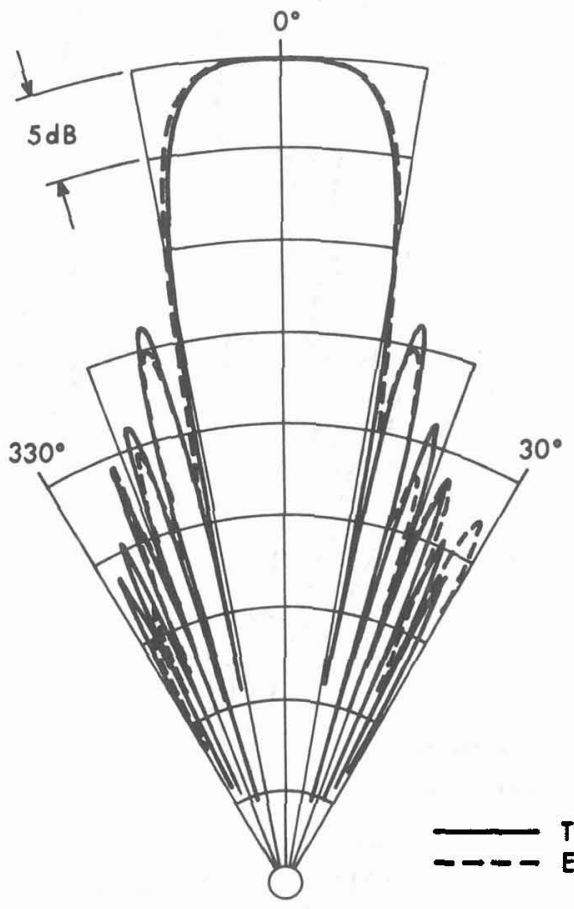

(a) Phase detector output pump transducer. The roles of the two transducers could be reversed so that the line transducer could be used either as a pump or as a rec ver. The array was suspended with an I-beam which could be rotated on a shaft. The electronic apparatus

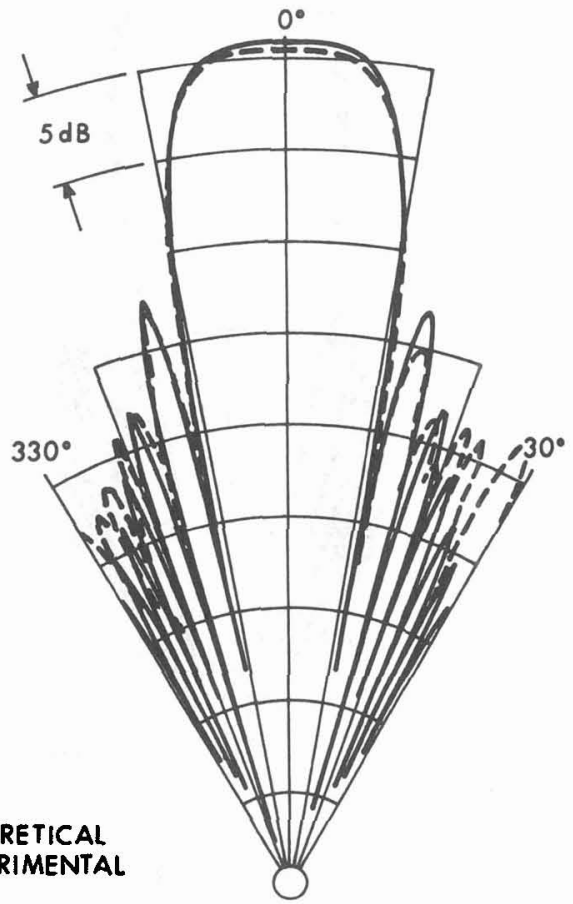

(b) AMPLITUDE DETECTOR OUTPUT

Fig. 4. - $5 \mathrm{kHz}$ Beam Pattern with the Pump Transducer Aligned with the Receiver. 
for these experiments was quite different from that used previousiy. Instead of a band-reject crystal filter, a phase demodulator and amplitude demodulator were used.

Parametric receiving array beam patterms were made with the phase demodulator and are shown in Fig. 4(a). The experimental results are compared with the theory of Eq. (18). In Fig. 4(b) we have the corresponding example for the amplitude detector. When the pump transducer was aligned, no essential difference was found between the beam pattern with the phase detector and the beam pattern with the amplitude detector. In Fig. 5(a) we show an example where the pump was misaligned so that the response of the pump signal was $3 \mathrm{~dB}$ down. The corresponding beam pattern for the output of the amplitude detector is shown in Fig. $5(b)$. We see that the beam pattern with the phase demodulator is essentially unchanged while the beam pattern for the amplitude demodulator is quite different. This difference occurs because the amplitude modulation term as expressed in Eq. (19) includes an asymmetrical component which is similar in am=plitude to the symmetrical component for our particular example. The beam pattern with a phase demo-

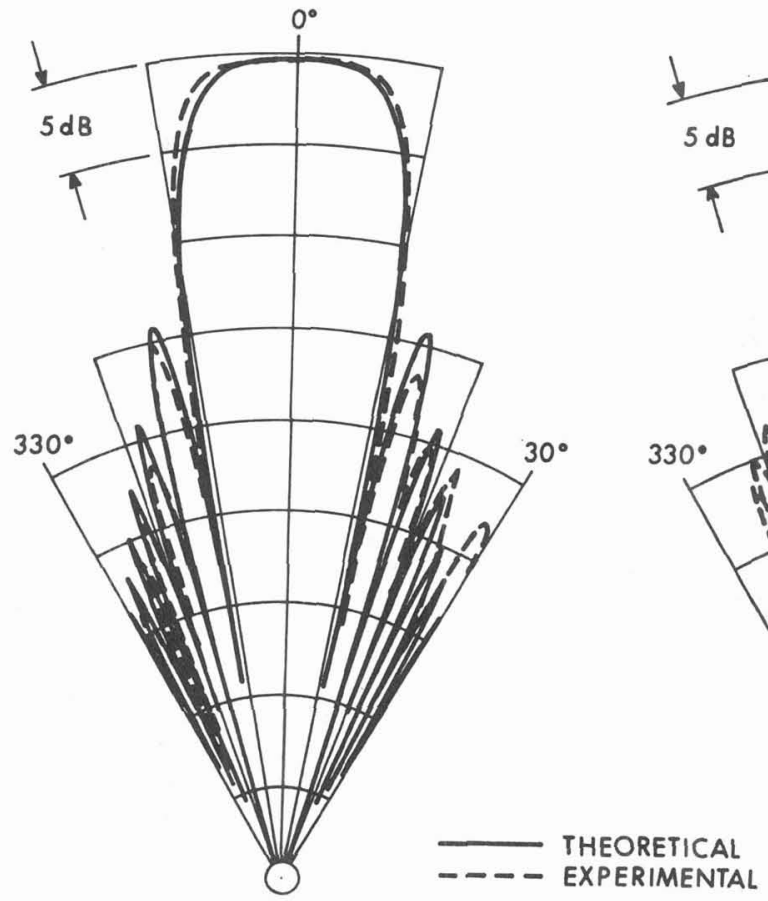

(a) PHASE DETECTOR OUTPUT

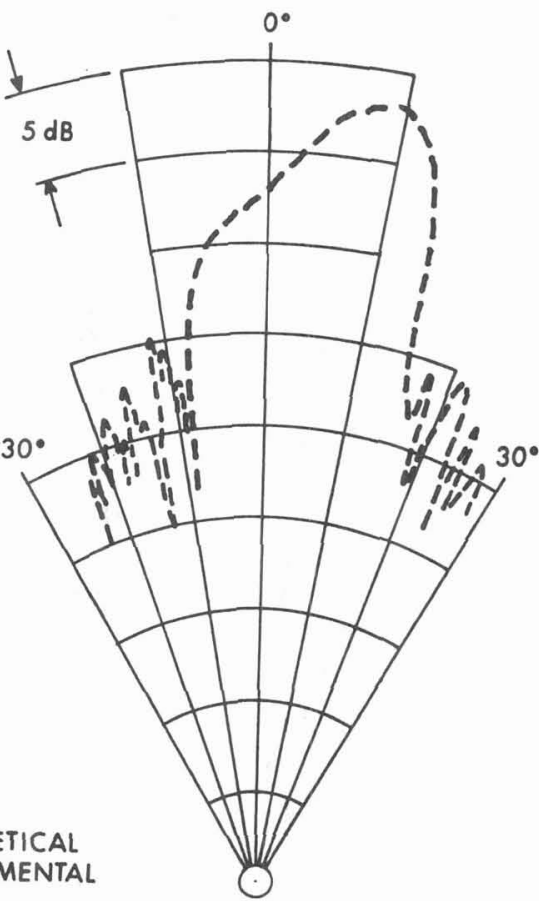

dulator remains nearly unchanged, while the beam pattern for the amplitude demodulator is dramatically changed when the pump transducer is misaligned.

Some difficulty in the experimental measurement of the amplitude modulation occurred due to a lack of flatness in the hydrophone response. Since the phase modulation components are considerably larger than the amplitude modulation components, the lack of flatness in the hydrophone response caused a small component of the phase modulation to appear as an amplitude modulation. Equalization of the hydrophone response would el iminate this problem. This effect increases the symmetrical component in the beam pattern.

CONCLUSIONS. - In this paper, we have derived the expressions for the phase and amplitude modulation components of a low frequency wave modulating a high frequency carrier in the example of the parametric receiving array. We have verified these expressions with experiments.

(b) AMPLITUDE DETECTOR OUTPUT

Fig. 5. $-5 \mathrm{kHz}$ Beam Pattern with the Pump Transducer Rotated to Its $3 \mathrm{~dB}$ Down Point. 


\section{ACKNOWLEDGMENTS}

The author would lice to express his appreciation to the many people at Applied Research Laboratories who assisted in the construction of equipment for the experiments and in the development of computer programs. This work was sponsored in part by the Office of Naval Research and the Naval Sea Systems Command.

\section{REFERENCES}

11/ J.J. Truchard, "The Detection of a Low-Frequency Plane Wave with a Parametric Receiving Array", Paper 2.12, presented at the 1973 Symposium on Finite Amplitude Wave Effects in Fluids, Copenhagen, Denmark.

/2/ J.J. Truchard, "Parametric Acoustic Receiving Array. I. Theory", J. Acoust. Soc. Am. 58, 1141-1145 (1975).
13/ J.J. Truchard, "Parametric Acoustic Receiving Array. II. Experiment", J. Acoust. Soc. Am. 58 , $1146-1150$. 